\title{
Probing Exciton Dynamics of Semiconducting Single-Walled Carbon Nanotubes Using Photon Echo Spectroscopy
}

\author{
Y.-Z. Ma ${ }^{1}$, M. W. Graham ${ }^{1}$, L. Valkunas ${ }^{2}$, S. M. Bachilo ${ }^{3}$, and G. R. Fleming ${ }^{1}$ \\ ${ }^{1}$ Department of Chemistry, University of California, Berkeley and Physical Biosciences \\ Division, Lawrence Berkeley National Laboratory, Berkeley, California 94720-1460, USA \\ E-mail: GRFleming@lbl.gov \\ ${ }^{2}$ Institute of Physics, Savanoriu Ave. 231, 02300 Vilnius, Lithuania and Theoretical \\ Physics Department, Faculty of Physics of Vilnius University, Sauletekio Ave. 9, build. 3, \\ 10222 Vilnius, Lithuania \\ ${ }^{3}$ Department of Chemistry and Center for Biological and Environmental Nanotechnology, \\ Rice University, 6100 Main Street, Houston, Texas 77005, USA
}

\begin{abstract}
Three-pulse photon echo peak shifts of single-walled carbon nanotubes were recorded at $975 \mathrm{~nm}$, showing an initial value of $26 \mathrm{fs}$, a dominant decay time of $59 \mathrm{fs}$ and an oscillatory frequency of $282 \mathrm{~cm}^{-1}$.
\end{abstract}

\section{Introduction}

Femtosecond optical spectroscopy has been applied to study ultrafast exciton population dynamics of semiconducting single-walled carbon nanotubes (SWNTs). The kinetics detected using transient absorption and time-resolved fluorescence depend strongly on excitation intensity owing to occurrence of exciton-exciton annihilation [1, 2]. This extremely rapid nonlinear process, in combination with the necessity of a time-independent annihilation rate to correctly describe the resulting kinetics, suggest involvement of coherent excitons [3, 4]. The three-pulse photon echo peak shift (3PEPS) technique has proved valuable for determining the time scales of dephasing processes that are coupled to an electronic transition and for separating homogeneous and inhomogeneous broadening [5]. In this contribution, we report what we believe to be the first 3PEPS experiment on a SWNTs-polymer composite film, in which the nanotubes are mainly individualized.

\section{Experimental Methods}

The 3PEPS technique employed here has been described extensively elsewhere [ 5 , 6]. In short, the light source was an optical parametric amplifier pumped by a 250 $\mathrm{kHz}$ Ti:sapphire regenerative amplifier, which generates $60 \mathrm{fs}$ pulses at $975 \mathrm{~nm}$. This wavelength was chosen in order to excite resonantly the lowest excitonic states $\left(E_{1}\right)$ of selected semiconducting SWNTs, namely, the $(6,5)$ and $(8,3)$ tubes. 
The sample was a $\sim 100-\mu \mathrm{m}$ thick film fabricated by individually dispersing HiPco nanotubes into a polymethylmethacrylate (PMMA) matrix. The total pulse energy incident on the sample was $\sim 4 \mathrm{~nJ}$.

\section{Results and Discussion}

Representative integrated photon echo profiles recorded at a population time $T=0$ fs are shown in Fig. 1a. The peak shift is obtained by fitting each of the profiles to a Gaussian function and then calculating half the difference between the maxima. The 3PEPS data obtained are plotted in Fig. $1 \mathrm{~b}$ as a function of $T$. The peak shift starts from an initial value of $\sim 26 \mathrm{fs}$ and decays rapidly to a persistent offset of about 8 fs within $200 \mathrm{fs}$. Weak but discernible oscillations are also evident.
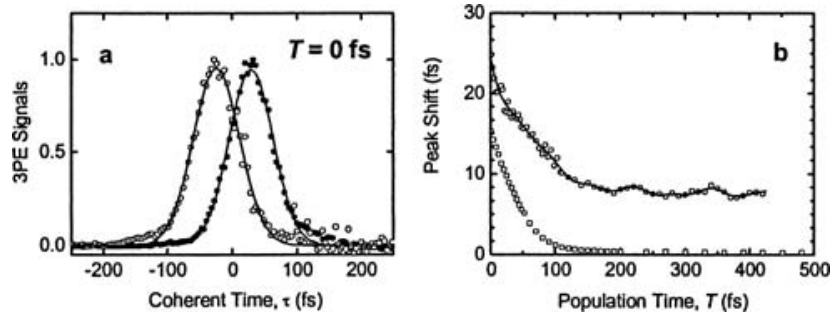

Fig. 1. (a) Normalized integrated photon echo profiles collected in the two phase matching directions $\mathbf{k}_{1}-\mathbf{k}_{2}+\mathbf{k}_{3}$ (open circles) and $-\mathbf{k}_{1}+\mathbf{k}_{2}+\mathbf{k}_{3}$ (filled circles) for the population time $T=0 \mathrm{fs}$. The solid lines are the fits to Gaussian functions. (b) 3PEPS data obtained for the SWNT/PMMA film (open circles) and a dichloroethane solution of IR26 (open squares). The solid line shows the fit to the 3PEPS data of the SWNT/PMMA film (see text).

Our preliminary analysis of the 3PEPS data shown in Fig. 1b involves a direct fit of the data to a sum of exponentials and damped oscillations:

$$
f(t)=\sum_{i=1}^{3} a_{i} \cos \left(\omega_{i} t+\phi_{i}\right) \exp \left(-t / \tau_{i}\right)+a_{4} \exp \left(t / \tau_{4}\right)+a_{5} \exp \left(-t / \tau_{5}\right)
$$

This analysis shows that three terms with corresponding amplitudes $a_{5}=20.6 \mathrm{fs}, a_{4}$ $=6.9 \mathrm{fs}$ and $a_{1}=4.4 \mathrm{fs}$ contribute significantly to the fit, whereas both $a_{2}$ and $a_{3}$ are an order of magnitude smaller than $a_{1}$. The time constants resolved for the three dominant components are $\tau_{5}=59 \mathrm{fs}, \tau_{4}=3.7 \mathrm{ps}$ and $\tau_{1}=53 \mathrm{fs}$, and the frequency of the oscillations associated with the $a_{1}$ component is $282 \mathrm{~cm}^{-1}$. While this analysis may appear arbitrary, the extracted oscillatory frequency is fairly close to the frequencies of the radial breathing modes of the selected $(8,3)$ and $(6,5)$ tubes, which are 298 and $307 \mathrm{~cm}^{-1}$, respectively [7].

The initial peak shift obtained for the SWNT/PMMA film is much larger (26 fs) than typically observed for dye molecules in organic solutions. As an example, we show in Fig. 1b (open squares) the 3PEPS data measured using IR26 dissolved in dichloroethane at the same excitation wavelength and intensity, which has an initial peak shift of 15 fs. Similarly large initial peak shifts have been widely observed for various photosynthetic pigment-protein complexes [6]. As found for 
molecular systems [6], this large initial peak shift is indicative of weak electronphonon coupling strength. On the other hand, the finite peak shift at long times $T$ suggests the presence of an inhomogeneous distribution of the $E_{1}$ transition frequencies in the ensemble of selected SWNTs. This is further supported by our recent finding of a similar peak shift at $T \approx 500$ fs from a solution sample.

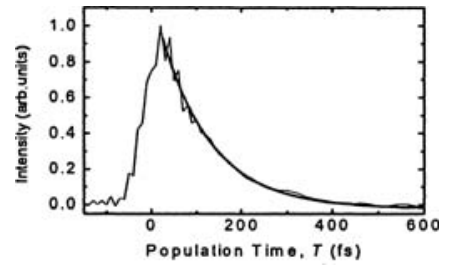

Fig. 2. Transient grating signal (thin line) and its fit (thick line).

Because the excitation intensity used for collecting the 3PEPS data is in the regime which induces exciton-exciton annihilation, it is necessary to verify that the time constants characterizing the 3PEPS data (Fig. 1b) are not due to the annihilation. To this end, we performed transient grating (TG) measurements under the same excitation intensity. Fig. 2 shows the TG signal, which can be satisfactorily fitted to a mono-exponential decay with a population decay time of $220 \mathrm{fs}$. As this decay time is clearly distinct from the time constants resolved from the 3PEPS data, it indicates that the population relaxation induced by the annihilation has no significant contribution to the temporal behavior of the 3PEPS signals.

\section{Conclusions}

Our 3PEPS data provide evidence of a weak electron-phonon coupling strength and the presence of an inhomogeneous distribution of the $E_{1}$ transition frequencies in the ensemble of the selected nanotube species. Detailed data simulation aiming at determining the electronic dephasing times and the amount of homogeneous and inhomogeneous broadening of absorption lines are currently in progress.

Acknowledgements. This work was supported by the NSF. L.V. thanks the Fulbright Foundation for financial support.

\section{References}

1 Y.-Z. Ma, J. Stenger, J. Zimmermann, S. M. Bachilo, R. E. Smalley, R. B. Weisman, and G. R. Fleming, J. Chem. Phys. 120, 3368, 2004.

2 L. F. Wang, G. Dukovic, E. Knoesel, L. E. Brus, and T. F. Heinz, Phys. Rev. B 70, 241403, 2004.

3 Y.-Z. Ma, L. Valkunas, S. L. Dexheimer, S. M. Bachilo, and G. R. Fleming, Phys. Rev. Lett. 94, 157402, 2005.

4 L. Valkunas, Y.-Z. Ma, and G. R. Fleming, Phys. Rev. B 73, 115432, 2006.

5 K. Ohta, M. Yang, and G. R. Fleming, J. Chem. Phys. 115, 7609, 2001.

6 R. Jimenez, F. van Mourik, J. Y. Yu, and G. R. Fleming, J. Phys. Chem. B 101, $7350,1997$.

7 S. M. Bachilo, M. S. Strano, C. Kittrell, R. H. Hauge, R. E. Smalley, and R. B. Weisman, Science 298, 2361, 2002. 